Linguistique, littérature, didactique

$177-178$ | 2018

Langage oral à l'école maternelle. Étude d'un corpus

homogène

\title{
Place des reformulations dans la construction d'un récit oral à l'école maternelle
}

The role of reformulations in French preschool pupils' elaboration of oral narratives

\section{Stéphanie Volteau et Victor Millogo}

\section{OpenEdition}

\section{Journals}

Édition électronique

URL : http://journals.openedition.org/pratiques/4232

DOI : $10.4000 /$ pratiques.4232

ISSN : 2425-2042

Éditeur

Centre de recherche sur les médiations (CREM)

Référence électronique

Stéphanie Volteau et Victor Millogo, «Place des reformulations dans la construction d'un récit oral à l'école maternelle », Pratiques [En ligne], 177-178 | 2018, mis en ligne le 20 juillet 2018, consulté le 01 mai 2019. URL : http://journals.openedition.org/pratiques/4232 ; DOI : 10.4000/pratiques.4232

Ce document a été généré automatiquement le 1 mai 2019.

(c) Tous droits réservés 


\title{
Place des reformulations dans la construction d'un récit oral à l'école maternelle
}

\author{
The role of reformulations in French preschool pupils' elaboration of oral \\ narratives
}

Stéphanie Volteau et Victor Millogo

\section{Introduction}

1 La réflexion menée dans cette contribution porte sur les formes des reformulations et leurs rôles dans des interactions verbales. Les reformulations participent de l'élaboration commune du discours. Cependant, elles interviennent de façon différente dans une conversation ordinaire, dans un dialogue spécifique comme l'échange entre une locataire et un conseiller juridique, qui constitue le corpus La dame de Caluire (Bange, 1987) ou dans une classe. En effet, les interactions en situation scolaire donnent lieu à une «communication inégale» (François, 1990) en raison de la nature différente des intervenants (un adulte/des enfants), de leur statut (un enseignant/des élèves) mais aussi de l'inégalité par rapport aux savoirs en jeu qui font l'objet de l'échange (Bruner, 1983).

2 Si les corpus oraux sont importants pour analyser et décrire les phénomènes interactionnels, on ne peut que souligner leur rareté comme l'exprimait déjà C. KerbratOrecchioni (1998, p. 52) :

" pour appréhender l'objet-langue, il faut d'abord s'intéresser à ses réalisations en milieu naturel, c'est-à-dire analyser de très près, sur la base d'enregistrement de données "authentiques", le fonctionnement d'échanges langagiers effectivement attestés. Or curieusement, il a fallu attendre en France les années 1980, pour voir certains linguistes recourir systématiquement à cette pratique descriptive, qui reste encore du reste minoritaire ».

3 Presque dix ans plus tard, G. Schmale (2010, p.13) souligne également « une pratique encore minoritaire en France ». 
Dans cette perspective, les corpus uniques de référence soumis à la communauté scientifique sont précieux parce qu'ils permettent des confrontations de points de vue sur des données enregistrées en situation écologique. On recense, par exemple, le corpus d'interaction verbale La dame de Caluire (Bange, 1987) se déroulant dans une institution entre deux personnes. Une recherche-action conduite à l'école primaire a donné lieu également à l'analyse d'un corpus unique portant sur des savoirs scientifiques et technologiques (Grandaty \& Turco, 2001). Et aujourd'hui, le corpus CLEA rendant compte de restitutions d'un même récit dans plusieurs classes de maternelle. C'est cet objet linguistique qu'est la reformulation orale au sein des interactions enseignant-élèves et élèves-élèves que nous nous proposons d'aborder ici. La reformulation est à considérer dans un processus interactif : «la reformulation n'est pas, pour nous, l'activité d'un seul locuteur, mais elle est produite interactivement, c'est-à-dire que plusieurs locuteurs peuvent contribuer à établir une relation de reformulation. C'est là même une qualité spécifique et essentielle des procédés de reformulation dans la communication orale » (Gülich \& Kotschi, 1987, p. 49).

5 Dans le cadre de cette contribution, l'intérêt que nous portons aux reformulations est double : d'une part, elles participent à l'apprentissage des formes linguistiques adaptées à travers l'étayage de l'environnement social (adultes, ici l'enseignant ou pairs dans l'interaction) et, d'autre part, elles favorisent l'intériorisation de l'utilisation du langage en contexte, en s'inscrivant dans un espace intersubjectif discursif (cf. Bernicot, Hudelot \& Salazar Orvig, 2006).

6 Nous articulerons notre réflexion autour d'une série de questions. Quelles formes linguistiques prennent les reformulations dans le corpus CLEA? Quelles fonctions remplissent-elles dans les interactions orales? Sont-elles plus ou moins nombreuses selon les séances analysées? Y a-t-il une influence du milieu scolaire? Est-ce que les reformulations participent aux apprentissages langagiers/linguistiques?

7 Après avoir présenté nos choix théoriques et méthodologiques, nous analyserons, tant sur le plan qualitatif que sur le plan quantitatif, les reformulations de l'enseignant et des élèves à partir de quelques extraits sélectionnés.

\section{Ancrages théoriques}

\section{Modélisations linguistiques : reformulations et paraphrases}

8 Lorsqu'elle définit la paraphrase, C. Fuchs (1982, p. 7) utilise le verbe reformuler : « on a coutume de dire qu'une phrase ou un texte $Y$ constitue une paraphrase d'une autre phrase ou d'un autre texte $\mathrm{X}$ lorsque l'on considère que $\mathrm{Y}$ reformule le contenu de $\mathrm{X}$; autrement dit, lorsque $\mathrm{X}$ et $\mathrm{Y}$ peuvent être tenus pour des formulations différentes d'un contenu identique, pour deux manières de dire la même chose ". S'inscrivant dans une approche d'analyse conversationnelle d'origine ethnométhodologique, É. Gülich et T. Kotschi (1987) analysent les reformulations dans une conversation entre un conseiller juridique d'une association de défense des locataires et une locataire. Ces auteurs formalisent la définition de C. Fuchs (1982) et considèrent que «la reformulation est une opération linguistique de la forme xRy, qui établit une relation d'équivalence sémantique entre un énoncé source $\mathrm{x}$ et un énoncé reformulateur $\mathrm{y}, \mathrm{R}$ étant le marqueur de reformulation " (Gülich \& Kotschi, 1987, p. 30). La relation d'équivalence sémantique se caractérise par la présence de traits dénotatifs et référentiels (ibid., p. 39). Trois catégories d'actes de 
reformulation sont distinguées : la paraphrase, la correction et le rephrasage. La notion de paraphrase repose sur une relation d'équivalence sémantique plus ou moins marquée entre l'énoncé source et l'énoncé reformulateur. Elle peut se présenter sous la forme d'une expansion, d'une réduction ou d'une variation. L'expansion se caractérise par un énoncé reformulateur plus étendu que l'énoncé-source. Elle se spécifie en deux souscatégories: a) l'explication définitoire qui comporte un trait définitoire dans l'énoncé reformulateur, et b) l'exemplification qui comprend l'apport d'aspects supplémentaires (traits référentiels) dans l'énoncé reformulateur. La réduction se caractérise par un énoncé reformulateur plus réduit que l'énoncé-source. Elle peut prendre la forme c) d'une dénomination, lorsque le terme spécifique est introduit après un énoncé source comportant un trait définitoire et $\mathrm{d}$ ) d'un résumé, lorsque l'énoncé reformulateur est plus réduit que l'énoncé-source. Enfin, la variation regroupe les reformulations qui ne sont ni des expansions ni des réductions. "Le ou les sémème(s) de l'énoncé-source ne sont ni décomposés dans un énoncé reformulateur plus étendu, ni comprimés dans un énoncé reformulateur plus restreint » (ibid., p. 42). Dans la correction, l'énoncé-source, considéré comme «fautif», se trouve alors partiellement ou totalement annulé par l'énoncé reformulateur. Le rephrasage se définit par « la répétition de la structure syntaxique et lexicale d'un énoncé » (ibid., p. 30).

9 À la suite d'É. Gülich et T. Kotschi (1987), M. de Gaulmyn (1987) analyse le même corpus, La Dame de Caluire, et propose de nouvelles catégories qui permettent d'affiner la typologie et de prendre en compte le fonctionnement spécifique de l'oral. Ainsi, M. de Gaulmyn, trouvant trop restrictive la catégorie de rephrasage lui préfère le terme de « répétition ». Parmi les répétitions, M. de Gaulmyn distingue les quasi-répétitions, les répétitions modifiées par addition ou soustraction partielle, les répétitions d'une amorce d'énoncé inachevée, les autorépétitions d'une répétition et les répétitions d'autodictée. Dans le cadre de la présente étude, la terminologie adoptée est très proche de celle proposée par M. de Gaulmyn (1987) en ce qui concerne les répétitions. Nous retenons pour l'étude des reformulations la notion d'invariant sémantique proposée dans la définition d'É. Gülich et T. Kotschi (1987).

\section{Interactions et construction des savoirs dans les interactions orales en situation scolaire}

10 La contribution de P. Bange et H. Kayser (1987, p. 279) sur le corpus La dame de Caluire a mis à jour les caractéristiques d'une interaction verbale: "nous dirons donc qu'une interaction verbale est une opération de co-construction par les participants d'un fragment de réalité sociale quotidienne sur la base d'un savoir commun ou conventionnel (c'est-à-dire social et non explicite) mis en œuvre en vue de la réalisation d'un objectif commun négocié compatible avec les intentions et préférences individuelles des interactants». Cette définition s'apparente tout à fait aux interactions en situation scolaire. On sait depuis les travaux en didactique sur le domaine de l'oral $^{1}$ que la verbalisation et les échanges langagiers participent à la dynamique des apprentissages (Nonnon, $1992 ; 1999$ ).

11 Une recherche-action coordonnée par M. Grandaty et G. Turco (2001, p. 415) a mis en évidence « le rôle central, de l'oral, de la construction des formulations au travers des reprises de formulation, de leurs types et de leur objet. Elle met aussi en avant la co- 
construction de la tâche langagière au croisement de la construction des savoirs et de la construction des discours ».

Les analyses croisées de chercheurs de différentes disciplines (sciences du langage et didacticiens) sur un corpus mathématique "polyèdres » à l'école élémentaire et un corpus anglais «mad cow» en lycée professionnel présentées dans l'ouvrage collectif dirigé par M.-C. Guernier, V. Durand-Guerrier et J.-P. Sautot (2006) montrent le rôle et la place des interactions orales dans la construction des apprentissages en contexte scolaire.

\section{Place des reformulations dans les interactions orales en situation scolaire}

Les reformulations sont constitutives des interactions didactiques entre l'enseignant et les élèves et participent de la structuration des savoirs et du développement du langage. D’après É. Nonnon (1990, p. 169-170), « il parait important, pour saisir dans sa dynamique le fonctionnement de la communication scolaire, de prendre en compte l'obligation institutionnelle et le devoir que les maîtres se donnent de faire progresser les enfants, c'est-à-dire de les aider à "mieux parler", ou "mieux réfléchir, mieux comprendre" à travers l'interaction verbale ».

D. Brixhe et A. Spécogna (1999, p. 10) tentent d'observer « comment les reformulations fournissent des indices significatifs de la contribution du maître à la découverte par les élèves d'une règle de dérivation des adverbes en "-ent" ». Les reformulations de l'enseignant témoignent de deux sortes d'activités de structuration des échanges: les activités de type interdiscursif ayant pour fonction d'assurer la cohérence des échanges, d'une part, et les activités de type intradiscursif permettant d'assurer la cohésion interne du discours de l'enseignant et de celui des élèves, d'autre part. Ces auteurs considèrent ces échanges verbaux comme des séquences verbales. D'autres travaux s'intéressent aux reformulations en tant qu'élément de l'expertise professionnelle (Garcia-Debanc \& Delcambre, 2001; Garcia-Debanc, Sanz-Lecina \& Margotin, 2001; Garcia-Debanc \& Laurent, 2003 ; Garcia-Debanc, 1998 ; 2007). La mise à l'épreuve du modèle linguistique d'É. Gülich et T. Kotschi (1987) permet de montrer quelques spécificités des interactions didactiques en observant les formes et les fonctions des reformulations, d'une part, et aussi de dégager quelques éléments de l'expertise professionnelle, d'autre part.

« Du côté des élèves, la reformulation témoigne des niveaux de formulation de la notion travaillée et constitue donc un indice intéressant du degré d'appropriation d'une notion. Du côté de l'expertise professionnelle de l'enseignant [...], la reformulation permet à l'enseignant de traiter la distance entre la formulation de certains élèves et la formulation pertinente » (Garcia-Debanc, Sanz-Lecina \& Margotin, 2001, p. 220-221).

La comparaison de plusieurs séances d'enseignement de domaines différents a mis en évidence l'usage préférentiel des reformulations par un enseignant débutant et un enseignant expérimenté (Volteau, Garcia-Debanc, 2008a et 2008b ; Volteau, 2009). Dans une autre étude, les reformulations différées dans une séquence portant sur l'écosystème dans une classe de CM2 mettent en relation construction des savoirs et appropriation de notions scientifiques (Volteau, 2015). 


\section{Choix méthodologiques pour l'analyse des reformulations dans le corpus CLEA}

16 Le corpus CLEA comprend douze séances/classes et pour les besoins de notre analyse nous avons opéré quelques choix.

\section{Sélection des séances}

17 Nous avons sélectionné six séances de GS afin d'observer ce qu'il se passe du point de vue des reformulations dans un même niveau de classe en contrastant les milieux scolaires retenus pour la transcription du corpus CLEA - à savoir - les quartiers sensibles, les Zones d'éducation prioritaire (ZEP) et les classes composées d'un public hétérogène.

Nous avons donc retenu les classes suivantes (Tableau 1) :

Tableau 1. Répartition des élèves en fonction de leur classe d'appartenance

\begin{tabular}{|c|c|c|c|c|}
\hline $\begin{array}{l}\text { Numéro de } \\
\text { séances }\end{array}$ & Niveau & $\begin{array}{l}\text { Nombre } \\
\text { d'élèves }\end{array}$ & $\begin{array}{ll}\text { Nombre } & \text { total } \\
\text { d'interventions } & \end{array}$ & $\begin{array}{l}\text { École- } \\
\text { quartier }\end{array}$ \\
\hline 2 & GS & 8 & 328 & ZEP \\
\hline 4 & GS & 10 & 242 & Hétérogène \\
\hline 8 & GS & 7 & 150 & Hétérogène \\
\hline 10 & GS & 12 & 185 & Hétérogène \\
\hline 11 & GS & 7 & 142 & Zone sensible \\
\hline 12 & GS & 10 & 125 & Hétérogène \\
\hline
\end{tabular}

19 Les séances 1 (MS/GS), 2 (MS) et 7 (MS) ont été écartées. Les séances 5 et 6 de niveau GS semblent être deux séances enregistrées dans la même classe avec la même consigne, à savoir « raconter l'histoire ». Aucune progression n'est observée.

20 Ces choix rendent plus homogène le corpus à observer.

\section{Problèmes méthodologiques dans la délimitation des reformulations}

21 Le repérage des reformulations puis leur identification n'est pas sans poser de problèmes. Nous en posons trois :

- Un problème d'identification de l'énoncé-source. L'énoncé-source provient-il de l'album ou d'un énoncé oral produit par l'enseignant ou un élève ? L'absence de transcription de la lecture de l'album à haute voix ou du contage nous contraint à nous focaliser exclusivement sur les seules interactions orales. Ce qui nous semble restrictif pour l'exploitation des reformulations. 
- La délimitation de la reformulation: où commence-t-elle? Où s'arrête-t-elle ? Sur quels indicateurs langagiers/linguistiques s'appuie-t-on? La tâche n'est pas aisée.

- La hiérarchisation des reformulations: on distingue celles qui permettent de ne pas interrompre la dynamique des échanges et qui relèvent plutôt d'un outil de communication et celles où le langage est un objet d'apprentissage dans lesquelles on repère une « information pertinente » au sens de M.-C. Guernier (2006, p. 19).

Nous avons choisi de nous focaliser sur les reformulations de l'enseignant qui participent de l'élaboration des savoirs, qui permettent une négociation de la signification et sur les reformulations des élèves qui manifestent des traces laissées dans le discours signalant une appropriation du lexique, une construction syntaxiquement complète, la compréhension d'un passage de l'histoire. Ces reformulations par les élèves peuvent être des reformulations de l'histoire (relation écrit-oral), des reformulations du discours de l'enseignant et d'un ou des élèves.

L'exemple suivant, extrait de la séance 12, illustre le type de reformulation qui nous intéresse ici.

Tableau 2. Exemple de reformulation paraphrastique

\begin{tabular}{|l|l|l|l|}
\hline Séance & $\mathbf{N}^{\mathbf{0}}$ & Loc & Interventions \\
\hline 12 & 105 & $\mathrm{M}$ & Anabelle \\
\hline 12 & 106 & Anabelle & il a glissé en dessous du portail \\
\hline 12 & 107 & $\mathrm{M}$ & comment on peut dire \\
\hline 12 & 108 & Anabelle & qu'il a: qu'il a: \\
\hline 12 & 109 & Juliette & faufilé/ il s'est faufilé \\
\hline 12 & 110 & Anabelle & voilà c'est ça que je voulais dire \\
\hline
\end{tabular}

L'énoncé 109 est une reformulation de 106 et porte sur le choix du lexique verbal. Nous sommes ici en présence d'une reformulation paraphrastique.

\section{Formes et fonctions des reformulations}

Notre objectif n'est pas de tenter un inventaire exhaustif des reformulations dans les séances sélectionnées du corpus CLEA mais de présenter leur fonctionnement dans une tâche de restitution de récit collectif à l'oral. Nous tenterons de montrer les formes et les fonctions que revêtent les reformulations tout en accordant une importance à l'élément déclencheur d'une reformulation (Gülich et Kotschi, 1987, p. 50). L'activité de reformulation peut être déclenchée par l'enseignant, un élève ou plusieurs élèves. Elle peut être du fait du locuteur lui-même dans un processus de réélaboration de son propre énoncé (autoreformulations) ou peut être produite par un autre interlocuteur dans un processus de coélaboration de l'énoncé de l'autre (hétéroreformulations) (Gülich et Kotschi, 1987, p. 50). 
Notre repérage des reformulations se réalise essentiellement dans des enchainements locaux.

Tous les exemples qui illustrent notre propos sont extraits des six séances retenues.

Le tableau $\mathrm{n}^{\circ} 3$ présente les catégories de reformulation qui feront l'objet d'une analyse qualitative. Nous mettons en évidence les formes et les fonctions de ces reformulations et précisons si elles sont déclenchées par l'enseignant ou les élèves.

Tableau 3. Catégories de reformulations repérées dans le corpus oral

\begin{tabular}{|c|c|c|c|}
\hline Catégories & Formes & Fonctions & $\begin{array}{l}\text { Structures des } \\
\text { interactions }\end{array}$ \\
\hline $\begin{array}{l}\text { Hétéro- } \\
\text { répétition }\end{array}$ & Répétition totale & Valider un énoncé d'élève & Auto-l'enseignant \\
\hline $\begin{array}{l}\text { Hétéro- } \\
\text { répétition }\end{array}$ & Répétition partielle & $\begin{array}{l}\text { Mise en évidence d'éléments } \\
\text { linguistiques importants }\end{array}$ & Hétéro - un élève \\
\hline $\begin{array}{l}\text { Hétéro- } \\
\text { répétition }\end{array}$ & Avec expansion & Apports de précisions & Auto - un élève \\
\hline $\begin{array}{l}\text { Hétéro- } \\
\text { correction }\end{array}$ & & Rectification & Auto - un élève \\
\hline $\begin{array}{l}\text { Hétéro- } \\
\text { répétition }\end{array}$ & $\begin{array}{l}\text { Répétition avec } \\
\text { modification }\end{array}$ & $\begin{array}{l}\text { Validation d'une réponse } \\
\text { d'élève et rectification }\end{array}$ & Hétéro - un élève \\
\hline Auto-répétition & $\begin{array}{l}\text { Répétition avec } \\
\text { modification }\end{array}$ & Manifester sa compréhension & Auto - un élève \\
\hline $\begin{array}{l}\text { Hétéro- } \\
\text { répétition }\end{array}$ & $\begin{array}{l}\text { Répétition avec } \\
\text { modification }\end{array}$ & $\begin{array}{l}\text { Réinvestir l'offre langagière de } \\
\text { l'enseignant }\end{array}$ & Hétéro - les élèves \\
\hline $\begin{array}{l}\text { Hétéro- } \\
\text { reformulation }\end{array}$ & $\begin{array}{l}\text { Reformulation } \\
\text { définitoire }\end{array}$ & $\begin{array}{l}\text { Aider à la compréhension en } \\
\text { contexte }\end{array}$ & Hétéro - un élève \\
\hline
\end{tabular}

\section{Répétition}

\section{Répétition totale}

L'exemple suivant illustre la catégorie des répétitions totales, il est extrait de la séance 2.

Tableau 4. Exemple de répétition totale

\begin{tabular}{|l|l|l|l|}
\hline Séance & $\mathbf{N}^{\mathbf{0}}$ & Loc & Interventions \\
\hline 2 & 156 & Elise & il est un peu méchant// \\
\hline 2 & 157 & $\mathrm{M}$ & alors explique ce un peu méchant \\
\hline
\end{tabular}




\begin{tabular}{|l|l|l|l|}
\hline 2 & 158 & Sandra & il veut pas partager son jardin// \\
\hline 2 & 159 & Elise & il est pas d'accord pour manger ses légumes// \\
\hline 2 & 160 & Sandra & et il défend son jardin \\
\hline 2 & 161 & M & $\begin{array}{l}\text { il défend son jardin qu'est-ce que vous auriez fait vous à la place de } \\
\text { monsieur Grégory/ Paulo }\end{array}$ \\
\hline
\end{tabular}

Nous parlons de répétition totale lorsqu'une structure linguistique présente dans l'énoncé-source est maintenue dans l'énoncé reformulateur. C'est le cas de l'énoncé S2-160 : et il défend son jardin et d'une partie de l'énoncé S2-161 : il défend son jardin. La suite de l'énoncé produit par l'enseignant montre un changement thématique ne nous permettant pas de l'inclure ici dans l'énoncé reformulateur.

Cette hétérorépétition a pour fonction de valider un énoncé produit par un élève. Elle est déclenchée par une demande d'explication de l'enseignant en S2-157 sur l'adjectif méchant ; énoncé lui-même déclenché par S2-156.

D'autres formes de répétition sont repérées dans le corpus CLEA. Il s'agit des répétitions partielles, des répétitions avec expansion et des répétitions avec modification d'un ou plusieurs éléments linguistiques que nous détaillerons ci-après.

\section{Répétition partielle}

Cette sous-catégorie se caractérise par le maintien d'une partie de l'énoncé-source dans l'énoncé-reformulateur. Voyons ici un extrait de la séance 11.

Tableau 5. Exemple de répétition partielle

\begin{tabular}{|l|l|l|l|}
\hline Séance & $\mathbf{N}^{\mathbf{o}}$ & Loc & Interventions \\
\hline 11 & 32 & $\mathrm{M}$ & Akem \\
\hline 11 & 33 & Akem & $\begin{array}{l}\text { et sa maman elle va donner une tisane de camomille pour pour pour se } \\
\text { calmer de sa journée parce que ses émotions lui reviennent }\end{array}$ \\
\hline 11 & 34 & $\mathrm{M}$ & ses émotions lui reviennent/ Julianne \\
\hline
\end{tabular}

L'enseignant répète les dernières parties de l'énoncé d'Akem. La sélection de ses émotions lui reviennent a pour fonction de valider et de mettre en évidence des éléments linguistiques importants à la compréhension du récit par l'élève et pour les élèves. Cette hétérorépétition est déclenchée par l'énoncé S11-33.

\section{Répétition avec expansion}

Voici un court extrait de la séance 4 : 
Tableau 6. Exemple de répétition avec expansion

\begin{tabular}{|l|l|l|l|}
\hline Séance & $\mathbf{N}^{\mathbf{0}}$ & Loc & Interventions \\
\hline 4 & 14 & Mélissa & $\begin{array}{l}\text { Capucine/ Neige et/// Neige ils allaient dans le pré et Pierre il } \\
\text { désobéissait à sa maman/ il allait dans le terr/ dans le }\end{array}$ \\
\hline 4 & 15 & Judicaël & dans le potager \\
\hline 4 & 16 & Mélissa & dans le potager de monsieur Grégory \\
\hline
\end{tabular}

Ici, l'énoncé-reformulateur est plus long que l'énoncé-source. Des éléments linguistiques sont ajoutés afin d'apporter des précisions sur le contexte du récit. L'hétérorépétition se réalise entre élèves et est déclenchée par S4-14 qui emploie dans le pré... dans le terr. Cet échange ternaire porte sur le choix des mots. Une rectification est apportée par Judicaël en S4-15: dans le potager qui semble plus approprié (mot provenant du texte original) que dans le pré... dans le terr. La proposition de Judicaël en S4-15 est réinvestie dans l'énoncé de Mélissa en S4-16.

\section{Répétition avec modification d'un ou plusieurs éléments linguistiques}

37 L'énoncé-source peut comporter à la fois des ajouts et des réductions. Dans l'exemple suivant extrait de la séance 2, l'hétérorépétition est initiée par Sandra qui demande une confirmation à l'enseignant

Tableau 7. Exemple de répétition avec modification

\begin{tabular}{|l|l|l|l|}
\hline Séance & $\mathbf{N}^{\mathbf{0}}$ & Loc & Interventions \\
\hline 2 & 33 & Sandra & $\begin{array}{l}\text { il est parti// quand il voyait le potager c'était un potager de rêve! il } \\
\text { mangeait/// des grosses radis géants? }\end{array}$ \\
\hline 2 & 34 & M & il mangeait des gros radis géants/ il mangeait/ \\
\hline
\end{tabular}

Cette demande de vérification est marquée par le point d'interrogation : il mangeait /// des grosses radis géants ?. L'hétérorépétition porte à la fois sur un énoncé syntaxique complet attestant la proposition de l'élève et à la fois sur une rectification portant sur le genre de l'adjectif grosses/gros.

D'autres éléments grammaticaux sont repérés dans les reformulations des élèves. Dans l'extrait ci-dessous, on repère une autorépétition avec modification en S2-215-S2-218.

Tableau 8. Exemple d'autorépétition

\begin{tabular}{|l|l|l|l|}
\hline Séance & $\mathbf{N}^{\mathbf{0}}$ & Loc & Interventions \\
\hline 2 & 215 & Sima & il écoute pas sa maman \\
\hline
\end{tabular}




\begin{tabular}{|l|l|l|l|}
\hline 2 & 216 & Sandra & et il fait pas comme ses frères et ses sœurs// \\
\hline 2 & 217 & Paulo & il est pas gentil \\
\hline 2 & 218 & Sima & l'écoute pas \\
\hline
\end{tabular}

Le syntagme nominal sa maman est remplacé par le pronom personnel $l$ '. Cette élève montre une maitrise des pronoms anaphoriques dans une restitution orale de récit. Cette autorépétition est auto-initiée par l'élève.

41 L'extrait suivant de la deuxième séance, porte sur le choix du verbe.

Tableau 9. Exemple d'hétérorépétition

\begin{tabular}{|l|l|l|l|}
\hline Séance & $\mathbf{N}^{\mathbf{0}}$ & Loc & Interventions \\
\hline 2 & 312 & Gaël & de sa journée/ il a failli se faire attraper \\
\hline 2 & 313 & M & et donc// \\
\hline 2 & 314 & Sandra & \\
\hline 2 & 315 & Gaël & mais il s'est pas fait avoir \\
\hline 2 & 316 & M & mais \\
\hline 2 & 317 & Elise & il s'est enfuyé \\
\hline 2 & 318 & Sandra & il a eu chaud \\
\hline 2 & 319 & M & il a failli se faire// dévorer \\
\hline 2 & 320 & Elise & déjà papa lapin il s'est fait dévorer par monsieur Grégory \\
\hline 2 & 321 & M & $\begin{array}{l}\text { déjà que le papa s'était fait dévorer par monsieur Grégory// on a parlé } \\
\text { partie certains donc Pierre à votre avis/ à l'avenir? }\end{array}$ \\
\hline
\end{tabular}

On observe : il a failli se faire attraper, il a failli se faire avoir, il ne s'est pas fait avoir, il s'est enfuyé, il a eu chaud, proposés successivement par les élèves. Ces termes, sans pour autant être rejetés, ne semblent pas convenir à l'enseignant qui offre en S2-319: il a failli se faire dévorer. Ce verbe est immédiatement réinvesti dans les propos d'Elise. L'hétéro-répétition S2-319-S2-320 est déclenchée par les énoncés précédents produits par les élèves.

D'autres reformulations ont été identifiées dans le corpus CLEA. Il s'agit des paraphrases et des reformulations définitoires. 


\section{Paraphrase et reformulation définitoire}

Paraphrase de type synonymie

L'analyse suivante porte sur un extrait de la séance 4.

Tableau 10. Exemple de paraphrase

\begin{tabular}{|c|c|c|c|}
\hline Séance & $\mathrm{N}^{\mathrm{o}}$ & Loc & Interventions \\
\hline 4 & 64 & Alice & il a il a tremblé \\
\hline 4 & 65 & M & oui Alice \\
\hline 4 & 66 & Alice & il tremblait il avait froid \\
\hline 4 & 67 & Thiébaud & et il a toussé \\
\hline 4 & 68 & Judicaël & et après il a fait aaatchoum \\
\hline 4 & 69 & Alice & il a fait aaatchoum \\
\hline 4 & 70 & $\operatorname{El}(s)$ & aaatchoum aaatchoum \\
\hline 4 & 71 & M & oui d'accord j'ai compris et ensuite \\
\hline 4 & 72 & Kendall & après \\
\hline 4 & 73 & M & Kendall \\
\hline 4 & 74 & Kendall & après monsieur \\
\hline 4 & 75 & $\operatorname{El}(\mathrm{s})$ & monsieur Grégory il l'a entendu \\
\hline 4 & 76 & M & oui Mélissa \\
\hline 4 & 77 & Mélissa & monsieur Grégory il l'a entendu et il l'a \\
\hline 4 & 78 & Thiébaud & il s'est retourné vers lui/// \\
\hline 4 & 79 & M & alors qu'est-ce qui se passe ensuite/ Siméon \\
\hline 4 & 80 & Siméon & il grelotte il fait atchoum \\
\hline 4 & 81 & $\mathrm{El}(\mathrm{s})$ & on l'a déjà dit \\
\hline 4 & 82 & $\mathrm{M}$ & Paulo \\
\hline
\end{tabular}

Nous considérons qu'il existe une relation d'équivalence sémantique de type synonymie entre trembler et grelotter. Mais on peut s'interroger sur la structure de la reformulation. Une analyse détaillée des tours de parole ne permet peut-être pas de considérer S4-64 il a 
tremblé comme l'énoncé-source de S4-80 il grelotte. Ce nouveau terme est introduit en réponse à la question de l'enseignant par un autre élève. Ce deuxième verbe grelotter est un réinvestissement du vocabulaire extrait du texte original : Pierre grelotte de peur et de froid et, tout à coup : «Atch... atch... atchoum!».

Dans la suite des échanges, on remarque que la proposition de l'élève, en S4-80, n'attire pas l'attention de l'enseignant qui aurait pu se saisir de cette offre lexicale. L'échange se clôture sur l'intervention de plusieurs élèves en S4-81.

\section{Reformulation définitoire}

Voyons l'exemple suivant extrait de la séance 2.

Tableau 11. Exemple de reformulation définitoire

\begin{tabular}{|l|l|l|l|}
\hline Séance & $\mathbf{N}^{\mathbf{0}}$ & Loc & Interventions \\
\hline 2 & 138 & $\mathrm{M}$ & donc deuxième étape on a dit qu'on parlait des \\
\hline 2 & 139 & Gaël & personnages// monsieur Grégory avec le papa il a fait un civet// \\
\hline 2 & 140 & Sandra & ça veut dire quoi personnages \\
\hline 2 & 141 & M & ça veut dire les gens qui sont dans une histoire// \\
\hline 2 & 142 & Alice & ou dans un livre// \\
\hline 2 & 143 & Elise & ou un dessin animé// \\
\hline
\end{tabular}

Ici, on peut identifier une reformulation définitoire de S2-140 à S2-143. Cette procédure définitoire est déclenchée en S2-139 lorsque l'élève emploie personnages dans son énoncé. Ce terme apparait comme un obstacle lexical pour Sandra en S2-140 qui s'interroge sur la signification de personnages. L'énoncé reformulateur se présente sous la forme d'une définition introduite par le marqueur ça veut dire. Ces éléments de définition proposés par l'enseignant sont complétés par deux autres élèves en S2-142 et S2-143. Cette pause lexicale permet d'aider une élève à accéder à la compréhension en contexte.

La définition peut ainsi être considérée comme le degré supérieur de l'activité métalinguistique pouvant se manifester dans une reformulation, ce qu'indique C. Fuchs (1982, p. 97) lorsqu'elle signale que l'«activité métalinguistique, dans sa dimension paradigmatique, concerne [...] la reformulation, depuis la pure et simple répétition jusqu'à la définition, en passant par tous les degrés d'interprétation ».

\section{Analyse quantitative des reformulations}

L'analyse quantitative des reformulations nous renseigne sur leur fonctionnement dans les situations d'interaction au sein des différentes séances/classes. En complément de l'analyse qualitative, le tableau 12 ci-dessous nous apporte des informations complémentaires sur plusieurs caractéristiques quantitatives des séances. Premièrement, nous constatons que le nombre de tours de paroles, représentant l'ensemble des 
interactions langagières de la classe, varie énormément d'une séance à l'autre: de 328 pour la séance 2 où les interactions sont les plus nombreuses, à 125 pour la séance 12 où les interactions sont les moins nombreuses. Ces variations d'une classe à l'autre dénotent d'importantes disparités au sein des groupes classes qui ne semblent pas liées à l'effectif des classes, globalement comparables. Quand on se centre sur la catégorie des énoncés de reformulation, on constate également d'importantes différences d'une classe à l'autre : 14 occurrences de reformulations pour la séance 10,16 occurrences pour les séances 11 et 12,33 occurrences pour la séance 8,43 occurrences pour la séance 4 , jusqu'à 80 occurrences pour la séance 2. Quant à la nature de ces énoncés de reformulation, hétéro et autorépétitions, elle met en évidence la place centrale occupée par les occurrences des hétérorépétitions, comparativement à celle des autorépétitions, et ce, quelle que soit la classe. Autrement dit, les situations dans lesquelles les segments linguistiques font l'objet de validation et de rectification par un autre interlocuteur sont plus nombreuses que celles qui résultent d'une explicitation de la part du même locuteur. Cela conforte l'idée selon laquelle ces reformulations constituent des indicateurs importants de la coélaboration, de la coconstruction des structures linguistiques au cours des interactions langagières orales en situation de classe.

Tableau 12. Aperçu des catégories de répétitions en fonction des séances et des classes

\begin{tabular}{|c|c|c|c|c|c|}
\hline & Reformulations & & $\begin{array}{l}\text { Nombre de } \\
\text { reformulations }\end{array}$ & $\begin{array}{l}\text { Nombre } \\
\text { d'élèves }\end{array}$ & $\begin{array}{ll}\text { Nombre de } \\
\text { tours de }\end{array}$ \\
\hline S2-ZEP & Hétérorépétitions & Autorépétitions & 80 & 8 & 328 \\
\hline $\begin{array}{l}\text { S4- } \\
\text { Hétérogène }\end{array}$ & 69 & 11 & 43 & 10 & 242 \\
\hline $\begin{array}{l}\text { S8- } \\
\text { Hétérogène }\end{array}$ & 31 & 12 & 33 & 7 & 150 \\
\hline $\begin{array}{l}\text { S10- } \\
\text { Hétérogène }\end{array}$ & 18 & 15 & 14 & 12 & 185 \\
\hline $\begin{array}{l}\text { S11-Zone } \\
\text { sensible }\end{array}$ & 8 & 6 & 16 & 7 & 142 \\
\hline $\begin{array}{l}\text { S12- } \\
\text { Hétérogène }\end{array}$ & 12 & 4 & 16 & 10 & 125 \\
\hline
\end{tabular}

51 La figure 1 ci-dessous représente les différentes formes de reformulations relevées dans les productions des élèves des différentes séances/classes étudiées. Nous avons exprimé en pourcentage les occurrences des différentes formes de reformulation pour permettre une comparaison directe entre les différentes séances/classes. Les analyses révèlent d'importantes disparités d'une classe à l'autre, d'une séance à l'autre, avec tout de même un certain nombre d'éléments communs à toutes les séances. Le premier résultat important concerne la forte proportion des répétitions avec modification. Cette forme de reformulation représente une proportion comprise entre $51,16 \%$ et $87 \%$ de l'ensemble 
des reformulations quelle que soit la séance ou la classe. Autrement dit, lorsqu'un énoncé est reformulé dans la classe, le nouvel énoncé reprend un ou plusieurs éléments de l'énoncé précédent tout en le modifiant, créant ainsi une progression dans la narration. La deuxième catégorie de répétitions correspond aux répétitions partielles; elle représente une proportion comprise entre $3,03 \%$ et $21,43 \%$. Cette forme de reformulation qui consiste à maintenir une partie de l'énoncé-source dans l'énoncéreformulateur semble se focaliser sur des éléments linguistiques essentiels à la compréhension du récit. Autrement dit, ces répétitions partielles jouent un rôle central de l'étayage du contenu du récit en cours d'élaboration collective.

Suivant les séances, les autres catégories de répétitions apparaissent plus ou moins : la proportion des répétitions totales est comprise entre $0 \%$ (aucune occurrence dans les séances 8 et 10) et $16,28 \%$ ( 7 occurrences pour la séance 4$)$; celle des répétitions avec expansion est comprise entre $0 \%$ (aucune occurrence dans la séance 11) et 16,28\% (7 occurrences pour la séance 4). Enfin, les paraphrasages (S2: 2 occurrences soit $2,5 \%$ et S4: 3 occurrences soit $6,98 \%$ ) tout comme les reformulations définitoires (S2: 2 occurrences soit $2,5 \%$; S8 : 1 occurrence soit 3,03\% et S12: 1 occurrence soit 6,25\%) sont plutôt très faiblement représentées et limitées à certaines séances.

Il ressort de ces analyses que plus de la moitié des interactions verbales au cours de la restitution $\mathrm{du}$ contenu $\mathrm{du}$ récit est réalisée à travers des répétitions avec des modifications d'un ou de plusieurs éléments linguistiques, et ce quelle que soit la séance/ classe. Toutefois, les paraphrasages et les reformulations définitoires semblent être plus complexes à manipuler pour les élèves de ces niveaux scolaires.

Figure 1. Répartition des différentes formes de reformulation en fonction des différentes classes

Image 20000009000070E400004F1FBEB0201A.wmf

Notre dernière analyse quantitative concerne la structure des interactions dans les reformulations. Nous avions noté que les reformulations pouvaient être déclenchées soit par l'enseignant, lorsque celui-ci permettait la validation d'un énoncé émis par un ou plusieurs élèves, soit par un élève à de multiples finalités (mise en évidence d'éléments linguistiques importants, apport de précisions, rectification, etc. - cf. Tableau 3). La figure 2 ci-dessous représente la proportion des interventions élèves-enseignant, enseignants-élèves, et élèves-élèves au cours de ces échanges.

Figure 2. Différentes catégories d'interactions dans les différentes classes

Image 20000009000071710000464D3BD4C434.wmf

L'analyse du fonctionnement des reformulations dans les échanges met en évidence des disparités importantes d'une séance à l'autre, avec néanmoins une constante quelle que soit la séance. Globalement, le déclenchement des éléments reformulateurs intervient plus fréquemment dans les interactions élèves-élèves (de 16,67\% à 93,55\%). Autrement dit, la reformulation d'un énoncé est déclenchée par un élève, puis cet énoncé peut faire l'objet d'une série d'opérations de négociation entre élèves. Ensuite, les interactions élèves-enseignant arrivent en deuxième position (de 6,45 \% à 61,54, suivant les séances) : nous interprétons ces interactions comme des indicateurs importants de la consolidation des échanges; dans ce cadre, le rôle de l'enseignant semble correspondre à celui de médiateur des échanges. Enfin, en dernière position, on retrouve les interactions 
enseignant-élèves (de $0 \%$ à 33,33\%), c'est-à-dire des interactions déclenchées par l'enseignant afin d'amener les élèves à réagir, de les solliciter à poursuivre le récit à partir d'éléments déjà présentés. Même s'il est difficile d'établir à priori des profils d'échanges dans la classe, il est tout de même intéressant de noter l'absence d'interactions dont l'enseignant est le déclencheur dans la plupart des classes hétérogènes ( $\mathrm{S} 4, \mathrm{~S} 10$ et $\mathrm{S} 12$ ) tandis que ces interactions représentent globalement un tiers des interventions dans les autres classes (S2 : $29 \%$; S8 : 33,33\% et S11 : 33,33\%). Toutefois cette interprétation doit être maniée avec prudence à cause de la constitution du corpus. On peut en effet s'interroger sur le nombre de relectures préalables à l'enregistrement de chacune des séances.

\section{Conclusion}

Notre description linguistique s'est attachée à rendre compte du travail conjoint de l'enseignant et des élèves dans les interactions orales. La mise à l'épreuve du modèle d'É. Gülich et Kotschi (1987) permet de mieux comprendre comment les élèves apprennent dans une situation de narration à l'école maternelle du point de vue des reformulations orales.

Les reformulations interviennent à différents moments des interactions verbales. Il n'est pas aisé de repérer les reformulations portant sur des moments d'apprentissage comme nous avons pu le montrer. Notre étude a tenté de sélectionner quelques formes de reformulations représentatives d'une production de récit oral à plusieurs. Nous avons pu observer que les répétitions occupent une place importante dans les interactions analysées. Ces constats sont à rapprocher des travaux de F. François, C. Hudelot et É. Sabeau-Jouannet (1984) qui ont montré le rôle central des répétitions dans les conduites de dialogue chez le jeune enfant.

Ce type d'analyse est essentiel en formation initiale et continue des enseignants afin de sensibiliser les enseignants à l'importance des gestes professionnels qui pour une grande majorité se réalisent dans et par le langage. La mise en œuvre des reformulations par l'enseignant/les élèves ne va pas de soi. Si certaines reformulations se réalisent de manière naturelle, ce n'est pas une généralité pour ce qui est des reformulations qui aident à la compréhension, qui valident un énoncé, qui explicitent un terme du métalangage etc. Celles-ci participent de la construction des connaissances à l'école.

\section{BIBLIOGRAPHIE}

BANGE, P. (éd.) (1987). L'analyse des interactions verbales. La dame de Caluire. Berne : P. Lang.

BANGE, P. \& KAYSER, H. (1987). « L'organisation d'une consultation. Approche théorique et empirique ». In : Bange, P. (éd.), L'analyse des interactions verbales. La dame de Caluire. Berne : P. Lang, p. 273-309. 
BERNICOT, J., HUDELOT, C. \& SALAZAR ORVIG, A. (2006). « Avant-propos. La reprise et ses fonctions ». La linguistique 2(42), p. 3-10. En ligne : https://www.cairn.info/revue-la-linguistique-2006-2page-3.htm.

BRIXHE, D. \& SPÉCOGNA, A. (1999). « Actes de reformulation et progression du savoir ». Pratiques 103-104, p. 9-27.

BRUNER, J. S. (1983). Le développement de l'enfant. Savoir faire, savoir dire. Trad. de l'anglais par M. Deleau. Paris : Presses universitaires de France.

DESCO (DIRECTION DE L'ENSEIGNEMENT SCOLAIRE). (2003). Didactiques de l'oral. Caen : CRDP BasseNormandie. En ligne : http://eduscol.education.fr/cid46413/sommaire.html.

FRANÇOIS, F. (1990). La communication inégale. Heurs et malheurs de l'interaction verbale. Neuchâtel : Delachaux et Niestlé.

FRANÇOIS, F., Hudelot, C. \& Sabeau-Jouannet, É. (1984). Conduites linguistiques chez le jeune enfant. Paris : Presses universitaires de France.

FUCHS, C. (1982). La paraphrase. Paris : Presses universitaires de France.

FUCHS, C. (1994). Paraphrase et énonciation. Gap/Paris : Ophrys.

GADET, F., LE CUNFF, C. \& TURCO, G. (1998). «L'oral pour apprendre ». Repères 17. En ligne : https:// www.persee.fr/issue/reper_1157-1330_1998_num_17_1?

sectionId=reper_1157-1330_1998_num_17_1_2242.

GARCIA-DEBANC, C. (1998). « Une argumentation orale dans une démarche scientifique au cycle 3 : ce que les reformulations nous disent des spécificités d'une interaction en situation scolaire ». Repères 17, p. 87-108 En ligne : https://www.persee.fr/doc/ reper_1157-1330_1998_num_17_1_2249.

GARCIA-DEBANC, C. (2007). « La reformulation orale : un élément de l'expertise professionnelle ». In : Talbot, L. \& Bru, M. (dirs), Des compétences pour enseigner. Entre objets sociaux et objets de recherche. Rennes : Presses universitaires de Rennes, p. 151-168.

GARCIA-DEBANC, C. \& DELCAMBRE, I. (dirs) (2001). « Enseigner l'oral ». Repères 24-25. En ligne : https://www.persee.fr/issue/reper_1157-1330_2001_num_24_1? sectionId=reper_1157-1330_2001_num_24_1_2378.

GARCIA-DEBANC, C., SANZ-LECINA, É. \& MARGOTIN, M. (2001). « Les compétences et les difficultés d'une enseignante débutante à gérer une situation d'oral dans le cadre d'activités scientifiques : étude de cas ». Repères 24-25, p. 201-236 En ligne : https://www.persee.fr/doc/

reper_1157-1330_2001_num_24_1_2378.

GARCIA-DEBANC, C. \& LAURENT, D. (2003). « Gérer l'oral en sciences : la conduite d'une phase d'émergence des représentations par un enseignant débutant ». Aster 37, p. 109-137. En ligne : http://documents.irevues.inist.fr/bitstream/handle/2042/8822/ ASTER_2003_37_109.pdf;jsessionid=BBB174D82A889DE7ECB288716B7EC3D2? sequence=1. GARCIA-DEBANC, C. \& PLANE, S. (coords) (2004). Comment enseigner l'oral à l'école primaire? Paris : Hatier.

GAULMYN, M. de (1987). « Actes de reformulation et processus de reformulation ». In : Bange, P. (éd.), L'analyse des interactions verbales. La dame de Caluire. Berne : P. Lang, p. 83-98.

GRANDATY, M. \& TURCO, G. (coords) (2001). L'oral dans la classe. Discours, métadiscours, interactions verbales et construction de savoirs à l'école primaire. Saint-Fons : INRP. 
GUERNIER, M.-C. (2006). « Introduction ». In : Guernier, M.-C., Durand-Guerrier, V. \& Sautot, J.-P. (dirs), Interactions verbales, didactiques et apprentissages. Recueil, traitement et interprétation didactiques des données langagières en contextes scolaires. Besançon : Presses universitaires de Franche-Comté, p. 15-28.

GUERNIER, M.-C., DURAND-GUERRIER, V. \& SAUTOT, J.-P. (dirs) (2006). Interactions verbales, didactiques et apprentissages. Recueil, traitement et interprétation didactiques des données langagières en contextes scolaires. Besançon : Presses universitaires de Franche-Comté.

GÜLICH, É. \& котSCHI, T. (1987). « Les actes de reformulation dans la consultation La dame de Caluire ». In : Bange, P. (éd.), L'analyse des interactions verbales. La dame de Caluire. Berne : P. Lang, p. 15-81. HALTÉ, J-F. (coord.) (1999). « Interactions et apprentissage ». Pratiques 103-104.

KERBRAT-ORECCHIONI, C. (1998). «La notion d'interaction en linguistique : origine, apports, bilan ». Langue française 117, p. 51-67. En ligne : https://www.persee.fr/doc/

lfr_0023-8368_1998_num_117_1_6241.

NONNON, É. (1990). « Est-ce qu'on apprend en discutant? ». In : François, F. et al., La communication inégale. Heurs et malheurs de l'interaction verbale. Neuchâtel : Delachaux et Niestlé, p. 147-212.

NONNON, É. (1992). « Un fantôme omniprésent ou un cadavre encombrant pour la didactique. Le rôle de la verbalisation et des échanges verbaux dans les démarches d'apprentissage ». Innovation 23/24, p. 9-22.

NONNON, É. (1999). «L'enseignement de l'oral et les interactions verbales en classe : champs de référence et problématiques ». Revue française de pédagogie 129, p. 87-131. En ligne : https:// www.persee.fr/doc/rfp_0556-7807_1999_num_129_1_1067.

SCHMALE, G. (2010). « Présentation. Interactions et Corpus Oraux ». Pratiques 147-148, p. 3-13. En ligne : https://journals.openedition.org/pratiques/1579.

VOLTEAU, S. (2009). Les reformulations orales dans des interactions didactiques au cycle 3 de l'école primaire. Formes et fonctions des reformulations d'une enseignante expérimentée et d'une enseignante débutante dans une classe de CM2. Thèse en sciences du langage : Université Toulouse 2 - Le Mirail. volteAu, S. (2015). « Analyse des reformulations dans les interactions orales : l'exemple d'une séquence portant sur l'écosystème en CM2 ». Corela HS-18. En ligne : https:// journals.openedition.org/corela/4045.

VOLTEAU, S. \& GARCIA-DEBANC, C. (2008a), « Les reformulations d'un enseignant dans quatre situations scolaires : influence de l'objet enseigné ». In : Schuwer, M., Le Bot, M.-C. \& Richard, É. (dirs), Pragmatique de la reformulation. Types de discours, interactions didactiques. Rennes: Presses universitaires de Rennes, p. 253-268.

VOLTEAU, S. \& GARCIA-DEBANC, C. (2008b). « Gérer les reformulations : un geste professionnel. Influence des objets enseignés sur les types de reformulation ». In : Bucheton, D. \& Dezutter, O. (dirs), Le développement des gestes professionnels dans l'enseignement du français. Un défi pour la recherche et la formation. Bruxelles : De Boeck, p. 191-212.

\section{NOTES}

1. Nous faisons référence à : J.-F. Halté (1999) ; F. Gadet, C. Le Cunff et G. Turco (1998) ; C. GarciaDebanc et I. Delcambre (2001) ; Desco (2003) ; C. Garcia-Debanc et S. Plane (2004). 


\section{RÉSUMÉS}

Les interactions orales constituent un espace privilégié pour les reformulations. En situation à visée didactique, les reformulations sont caractéristiques des processus d'apprentissage/ d'acquisition de la langue. Elles participent en effet de l'élaboration commune du discours. En validant, en corrigeant ou en problématisant les énoncés des élèves, l'enseignant participe à la construction des savoirs, à la structuration des connaissances et au développement langagier de ses élèves. La présente contribution se propose, dans une approche descriptive du corpus commun CLEA, de rendre compte des reformulations de l'enseignant et des élèves ainsi que de leurs fonctions dans les interactions orales à partir de restitutions de récit menées dans six classes de grande section de maternelle (4-5 ans).

Oral interactions constitute a privileged space for reformulations. In didactic situations, reformulations are characteristic of the language learning/acquisition processes. They contribute to the co-construction of discourse. The teacher participates in the construction and structuring of his/her pupils' knowledge and in the development of their linguistic skills. He/she does so by validating, correcting or problematizing the students' statements. Our research falls within the framework of a descriptive approach of the common CLEA corpus and aims to account for the teachers' and the pupils' reformulations and their functions in oral interactions. We thus analyzed a set of narratives provided by preschool pupils aged 4-5 from 6 different classes.

\section{INDEX}

Keywords : verbal interactions, reformulations, language development, repetitions, learning Mots-clés : interactions verbales, reformulations, développement du langage, répétitions, apprentissage

\section{AUTEURS}

\section{STÉPHANIE VOLTEAU}

Université de Poitiers, FORELL, EA 3816, ESPÉ de l'Académie de Poitiers, F-86073, France

\section{VICTOR MILLOGO}

Université de Poitiers, CeRCA, UMR CNRS 7295, ESPÉ de l'Académie de Poitiers, F-86073, France 